\title{
Analysis of camphor and camphoric acid
}

\section{Liebig}

To cite this article: M. Liebig (1832) Analysis of camphor and camphoric acid, Philosophical Magazine Series 2, 11:62, 149-150, DOI: $\underline{10.1080 / 14786443208647703}$

To link to this article: http://dx.doi.org/10.1080/14786443208647703

册 Published online: 25 Jun 2009.

Submit your article to this journal $x$

Џ Article views: 2

Q View related articles $₫$ 
lain furnace. More lately Gmelin attributed this reduction per se to the presence of gaseous oxide of carbon in the furnace, and assigned the same cause for the reduction of oxide of iron in a porcelain furnace observed by Proust. In fact, it appears contradictory that a metal which oxidizes so readily as nickel, by calcination in the air, which burns in oxygen gas with disengagement of light, and is even susceptible of spontaneous combustion at common temperatures when it is much divided, should be reduced from its oxide merely by the action of a strong heat. Nevertheless, it does not appear that direct experiments have been made on this subject, although it has often been remarked that during the reduction of nickel in porcelain furnaces, without the use of charcoal, less metal was always obtained when the crucible was well closed. The following experiments will undoubtedly suffice to justify the opinion, that the reduction of nickel per se is only apparent, and that it is in fact due to the oxide of carbon disengaged in the furnace.

Two equal portions of oxide of nickel, equally pure and treated in the same manner, were placed in two crucibles and exposed together to the most intense heat of a porcelain furnace. One of the crucibles was lightly covered, whilst the other was covered internally and externally, with a coating which was vitrifiable by heat; it was not only covered with a smaller crucible, rendered equally impervious to air, but it was put into another of larger dimensions, also covered with a second, and well luted. The interval was filled with fine sand. When these crucibles were taken out of the furnace after calcination, that is to say, after a fire of eighteen hours, there was found in that which had been lightly covered nearly five grammes of white metallic malleable nickel, incrusted with much fused and unreduced oxide. On the other hand, in the coated crucible, mere fused oxide was found, or containing only small globules of metal, of the size of a pin's head; and their reduction merely proves that it is impossible to render a clay crucible impermeable to gases, in a violent and long-continued heat. -Ann. de Chim. et de Phys. xlviii. p. 257.

ANALYSIS OF CAMPHOR AND CAMPHORIC ACID. BY M. LIEBIG. Camphor, taking the mean of two analyses, appeared to consist of

$$
\begin{aligned}
& \text { Hydrogen............ } 9702 \\
& \text { Oxygen.............. } 8.535 \\
& \text { Carbon............. } 81 \cdot 763 \\
& 100 \cdot 000
\end{aligned}
$$

According to Bouillon-Lagrange the camphorates of soda, potash and barytes are so little soluble in water that one part requires from 200 to 300 parts for solption, whilst according to Brandes these salts are deliquescent. According to $\mathrm{M}$. Liebig, these differences depend upon the different kinds of camphoric acid on which the experiments were made.

When camphor is treated with concentrated nitric acid, a yellow liquid 
liquid is formed; by long continued digestion, there is obtained on cooling a large quantity of white opaque crystals, which when boiled in water yield a smell of camphor. These crystals are the camphoric acid of Lagrange, which gives with all bases insoluble or slightly soluble salts.

These crystals are a chemical compound of camphor and camphoric acid, which may be prepared directly by dissolving camphor in camphoric acid melted at a very low heat.

If this substance be treated a second time with concentrated nitric acid, more transparent crystals of camphoric acid are obtained, which give precisely the salts described by Brandes: when a boiling solution of this camphoric acid was poured into one of acetate of lead, camphorate of lead was formed, 100 parts of which gave 67.7 of sulphate of lead; analysis, however, rendered it probable that the camphoric acid still retained some camphor in combination; it was therefore again treated with nitric acid, and the action was continued until no smell of camphor was perceptible when the acid was boiled in water; 1105 parts of camphorate of lead gave 760 of sulphate. By analysis it yielded

$$
\begin{aligned}
& \text { Hydrogen ............ } 6.981 \\
& \text { Oxygen............ } 36.852 \\
& \text { Carbon............. 56.167 } \\
& 100 \cdot 000 \\
& \text { Ibid. xlvii. p. } 95 .
\end{aligned}
$$

ON LACTIC ACID. BY BERZELIUS.

Berzelius having ascertained that lactic acid is not acetic acid and an animal matter analogous to the sulphovinic acid, nor a compound of acetic acid and non-volatile animal matter, gives the following methods for obtaining it in purity : the acid alcoholic extract obtained from milk or meat is to be dissolved in alcohol; the solution is to be mixed with one of tartaric acid in alcohol as long as precipitation takes place; the mixture is to remain for 24 hours in a cold place, in order that all the double tartrate which it contained may deposit. The alcohol is to be evaporated, the residue dissolved in water, and well powdered carbonate of lead added to it as long as it dissolves, and until the solution has a sweet taste; it is then to be treated with animal charcoal, and afterwards with sulphuretted hydrogen gas to remove the lead. The liquor is to be evaporated until all the sulphuretted hydrogen is expelled, and then mixed with well washed, moist, recently precipitated protohydrate of tin, with which it is to remain for several days, and sometimes to be stirred. The sublactate of tin formed is to be well washed and decomposed by sulphuretted hydrogen, and the lactic acid resulting is the purest which Berzelius has hitherto obtained: in this process much acid however remains in solution, and is lost.

Another method is to saturate the free acid of the alcoholic extract by means of carbonate of soda or potash; the solution is to be dried, and the mass heated on a sand-bath until it fuses, becomes 